\title{
Optical response of WO3 nanostructured thin films sputtered on different transparent substrates towards hydrogen of low concentration.
}

\begin{abstract}
The gasochromic response of WO3 nanostructured films coated with a catalytic Pt or Pd layer on different transparent substrates upon exposure to $\mathrm{H} 2$ gas was investigated. WO3 nanostructured films with $500 \mathrm{~nm}$ thickness were coated with a $25 \AA$ thick Pt or Pd layer. The films were prepared on quartz, glass, indium-doped tin oxide (ITO) and fluorine-doped tin oxide (FTO) conductive glass. The nanostructured WO3 was deposited by RF magnetron sputtering, and the Pt or Pd layer was deposited by DC sputtering. Characterization of the film revealed that the WO3 was deposited as nanoscale grains of varied size depending on the substrate. WO3 grains on quartz and glass were 30-40 nm in size. WO3 grain sizes on ITO and FTO were 40-60 nm and $300-500 \mathrm{~nm}$, respectively. The WO3 films were observed to show strong gasochromic response, with absorbance changes measured in the Vis-NIR (500-1100 nm) range. The cumulative absorbance response towards $\mathrm{H} 2$ is the highest and more stable for Pd/WO3 films on quartz, glass and ITO, compared to the FTO substrate. The gasochromic effect was also stronger in $\mathrm{Pd} / \mathrm{WO} 3$ films compared to Pt/WO3 films.
\end{abstract}

Keyword: Tungsten trioxide; Hydrogen; Optical sensing; Absorbance; Transparent substrates; RF sputtering 